\title{
Aplicación de Design Thinking para la sistematización de procesos artísticos en el alumnado de Secundaria
}

\section{Application of Design Thinking for the systematic process of artistic processes in Secondary students}

\author{
Nora Ramos Vallecillo ${ }^{1}$ \\ ${ }^{1}$ Universidad de Zaragoza. noramos@unizar.es
}

Recibido: $14 / 11 / 2019$

Aceptado: 21/4/2020

Copyright (C)

Facultad de CC. de la Educación y Deporte. Universidad de Vigo

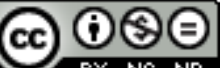

Dirección de contacto: Nora Ramos Vallecillo Facultad de Educación Despacho 4070

Universidad de Zaragoza

C/ Pedro Cerbuna, 12

50009 ZARAGOZA

\begin{abstract}
Resumen
Este trabajo presenta los resultados de una investigación centrada en el análisis de la influencia que tiene la práctica del Design Thinking en el desarrollo de los procesos artísticos en estudiantes de Secundaria. El estudio tomó como eje de indagación un proyecto realizado dentro del área de Educación Plástica, Visual y Audiovisual. Se utilizó un estudio de caso como metodología de la investigación y el análisis de documentos, observación participante, entrevistas y grupos de discusión como estrategia de recogida de información. Los resultados alcanzados permiten constatar un aprendizaje sustentado en el hacer, explorar y experimentar, con la finalidad de aprender a aprender. A partir de los hallazgos obtenidos se concluye que el Design Thinking conforma un marco metodológico pertinente para la adquisición y desarrollo de competencias.
\end{abstract}

\section{Palabras clave}

Design Thinking, Educación Artística, Competencias

\begin{abstract}
This article presents the results of an investigation centered on the analyzing of the influence of the practice of Design Thinking practices on the development of artistic processes in high school students. The study took as its axis of inquiry a project carried out within the area of Arts Education. The case study was used as a research methodology and documents analysis, observation, interviews and focus groups as information gathering strategies. The results achieved allow us to verify a learning based on doing, exploring and experimenting, in order to learn to learn. Based on the findings obtained, we conclude that Design Thinking forms a relevant methodological framework for the acquisition and development of competence.
\end{abstract}

\section{Key Words}

Design Thinking, Arts, Competencies 


\section{INTRODUCCIÓN}

Desde finales del siglo XX el mundo de la educación ha sufrido una transformación paulatina en relación con las metodologías de trabajo en el aula. En los últimos años los modelos de enseñanza centrados en el alumnado se han introducido con gran fuerza como modelo educativo.

Los cambios sociales junto a la repercusión de los avances tecnológicos, con un crecimiento de la disponibilidad de dispositivos móviles y la universalización del acceso a internet, han ocasionado un gran impacto en la educación y en las formas de aprendizaje. Este cambio de paradigma en el sistema educativo ha originado la necesidad de quitar el énfasis en la adquisición de contenidos para generar habilidades que posibiliten el desarrollo integral de competencias. La sociedad demanda que el alumnado sea capaz de gestionar el conocimiento para resolver situaciones y problemas creativamente y no sea un mero reproductor de información.

En la actualidad los estudiantes aprenden activamente, en entornos fuera del aula, por medio de aprendizajes en red... todos estos tipos de actividades se han convertido en formas de aprendizaje en expansión, y por ello debemos establecer nuevas maneras de enseñar. Estos cambios en la sociedad del conocimiento han hecho que sea necesario modificar las prácticas docentes, que afectan tanto a la planificación, como a aspectos curriculares y metodológicos.

La enseñanza del arte en la escuela tiene una trayectoria en la que constantemente se ha venido cuestionando su importancia (Calaf y Fontal, 2010). La educación de las artes visuales ha sido concebida durante mucho tiempo como una asignatura para el desarrollo artístico de aquellos alumnos que poseían capacidades innatas y por eso se llevaba a la práctica por medio de la realización de ejercicios. La educación plástica ha estado limitada en sus capacidades educativas debido a la idea del genio creador (Hernández, Jódar y Marín, 1991; Fontal, Gómez y Pérez, 2015). Este ha sido uno de los problemas con los que ha tenido que lidiar la educación artística a lo largo de su historia. El ampararse en la libertad de la genialidad del artista sin el sustento de una metodología, es lo que hace que se considere una asignatura sin criterios establecidos.

Hernández (1995) destaca que, en España, durante muchos años, la tendencia fue centrar la educación artística en la expresividad de los estudiantes, el conocimiento de un lenguaje y la realización de un producto final. Esta situación restringía las posibilidades educativas de la asignatura haciendo que se centrara principalmente en el desarrollo de destrezas y habilidades manuales, donde el resultado final era, en la mayoría de los casos, un dibujo realizado a partir de unos conceptos desarrollados en una unidad didáctica. Las actividades estaban circunscritas a unos contenidos concretos, desconectados de las formas de aprendizaje de los alumnos y alumnas. Esta metodología carecería de dicha visión globalizada y preparación integral de cara a su futura aplicación en un entorno real orientado al mundo laboral vigente.

Acaso y Megías (2017) consideran que la educación artística del pasado ha sido entendida como instrucción en vez de como educación, como actividad y no como acción, como una sucesión de actividades inconexas en lugar de como un programa. La educación artística, que constituía una propuesta coherente para los años sesenta, no tiene sentido hoy.

La metodología más extendida en las clases de educación artística es la que sitúa al libro de fichas como eje esencial, acompañada de momentos de realización de objetos 
decorativos que poco tienen que ver con experiencias creativas (Fontal, Marín y García, 2015). Los trabajos realizados en educación plástica no deben limitarse a la adquisición de destrezas manuales, deben desarrollar en los alumnos capacidades como la creatividad, la autoexpresión y la percepción. Hay que reivindicar una enseñanza de las artes como un área relacionada con el conocimiento, con el intelecto, con los procesos mentales y no sólo con los manuales (Acaso, 2009).

En la actualidad nos encontramos con cambios a nivel pedagógico y social. Nos hallamos en la era de la imagen y, como consecuencia de ello, las últimas tendencias artísticas están abiertas a todo tipo de materiales, conceptos e ideas. La formación en artes visuales debe ser coherente con la diversidad artística actual, y por ello debe buscar métodos que favorezcan la adquisición de modos de pensamiento. Estos cambios hacen que sea necesaria la revisión de algunos planteamientos metodológicos frente a la necesidad de dar respuestas. Esta realidad educativa nos conduce a diseñar metodologías y a seleccionar contenidos desde una perspectiva radicalmente distinta (Acaso y Megías, 2017). Dentro del área de Educación Plástica, Visual y Audiovisual (en adelante EPVyA) se deben establecer unas pautas didácticas para ensalzar el aprendizaje artístico y la comprensión de la cultura visual. Hay una clara necesidad de educar a los adolescentes para que sean capaces de construir conocimiento crítico con el que comprender la realidad visual que les rodea. Por ello, debemos proporcionar a nuestro alumnado las herramientas necesarias para la comprensión de estos medios en toda su amplitud.

Al igual que Nisbet y Shucksmith (1980) se considera que una de las finalidades de la educación artística debe ser la de enseñar a utilizar estrategias de aprendizaje y de resolución de problemas, teniendo en cuenta que, tomando su definición como referencia, las estrategias de aprendizaje son los procesos que sirven de base a la realización de las tareas intelectuales. Por tanto, como destacan Calaf y Fontal (2010) el arte requiere de un cuerpo teórico, metodológico y experimental específico que conformen la disciplina de educación artística.

Si tenemos en cuenta la EPVyA como una asignatura curricular debemos tratarla como tal y buscar un método que aporte esa libertad necesaria para el área, pero claramente determinada por una praxis estudiada y contrastada. La metodología no es una atadura, es lo que aporta la libertad de saber que estamos realizando la labor docente de manera adecuada y sustentada por estudios que así lo corroboran. En la actualidad dentro del área hay una clara necesidad de encontrar un modelo metodológico integrador que, además de los objetivos desarrollados por medio de la práctica, tenga en cuenta el momento social cambiante y globalizador que estamos viviendo.

El aprendizaje activo, del que forma parte el Design Thinking, se caracteriza por estimular al alumnado a generar sus propias soluciones, aplicando conceptos y teorías a diversas situaciones. Aprender con sentido, a partir de lo que se conoce, de forma activa y con tareas reales, serán la garantía de un aprendizaje auténtico (Pellicer, Álvarez y Torrejón, 2013).

Coll (1978) señala la actividad del estudiante como un aspecto fundamental del proceso educativo, y distingue diferentes tipos de actividad: la motriz o verbal, la actividad de seguimiento de instrucción, la realizada mediante enseñanza programada y la actividad aceptando un objetivo. El aprendizaje activo estaría ubicado dentro de este 
último tipo, donde la importancia de la actividad está en la adquisición del conocimiento.

Los nuevos modelos educativos basados en la actividad demandan que los docentes transformen su rol de expositores del conocimiento al de guías del aprendizaje, y los estudiantes, de espectadores del proceso de enseñanza, al de integrantes participativos, propositivos y críticos en la construcción de su propio conocimiento (Sánchez, Brahim y López, 2013). El alumnado organiza y estructura verdaderamente su propio trabajo, lo que implica un cambio considerable de la actitud y función del docente. El protagonista del aprendizaje es el propio aprendiz que debe desarrollar actitudes de autonomía en su aprendizaje.

Los docentes deben enseñar al estudiante a aprender y ayudarle a construir esquemas mentales. Su papel es el de acompañar, guiar y evaluar a las alumnas y alumnos cuando es necesario. Debe modelar y guiar a los estudiantes para manejar apropiadamente sus tareas, siendo orientador del aprendizaje y de los procesos, y dejando que el alumnado adquiera autonomía y responsabilidad en su aprendizaje.

\section{FUNDAMENTACIÓN TEÓRICA}

El Design Thinking es un enfoque colaborativo, de resolución de problemas y centrado en el ser humano (Aflatoony, Wakkary y Neustaedter, 2017). Es una manera de resolver problemas reduciendo riesgos y aumentando las posibilidades de éxito y usa diferentes tipos de inteligencia como la integral, emocional y experimental (Serrano y Blázquez, 2014).

El Design Thinking tiene 5 etapas o fases fundamentales (Steinbeck, 2011; Portnoy, 2018):

- Empatizar con los usuarios. Empatizar requiere tanto compromiso activo como reflexión y observación pasiva. En las aulas, empatizar significa identificar posibles limitaciones físicas o determinar problemas locales que afectan a la comunidad, que los estudiantes están motivados para resolver. Hay que entender los problemas, necesidades y deseos de los usuarios implicados en la solución que estamos buscando. Independientemente de qué estemos desarrollando, siempre comportará la interacción con personas. Satisfacerlas es la clave de un resultado exitoso.

- Definir el problema. El objetivo de esta segunda etapa es sintetizar los datos para determinar si surgen patrones. Estos patrones ayudan a los diseñadores a definir problemas. Los estudiantes pueden trabajar de manera colaborativa o independiente para definir problemas basados en sus observaciones. El trabajo en equipo pone en valor la capacidad de los individuos de aportar singularidad.

- Generación de datos. En esta etapa los datos recopilados son una fuerza impulsora en el aula. Es hora de idear o utilizar los datos mientras se identifica con el usuario para encontrar posibles soluciones a los problemas identificados. Durante el proceso se desarrollan técnicas con un gran contenido visual y plástico. Esto hace que pongamos a trabajar tanto nuestra mente creativa como la analítica, dando como resultado soluciones innovadores y a la vez factibles. Todo ello bajo una atmósfera en la que se promueve lo lúdico. Se trata de disfrutar durante el proceso, 
y gracias a ello, llegar a un estado mental en el que demos rienda suelta a nuestro potencial.

- "Prototipar" una solución. La etapa de ideación presenta una gran cantidad de innovaciones potenciales que piden ser prototipadas y probadas con los usuarios. Lo emocionante de la creación de prototipos es que los estudiantes toman ideas que abarcan todo el nivel de impacto y factibilidad, y se ponen a trabajar para crear una solución. El objetivo con el prototipo es avanzar con posibles soluciones que puedan tener impacto y viabilidad mientras se usan menos recursos. Este proceso garantiza que los estudiantes se centren en el diseño y no en la perfección y les permite ver que el fracaso no es algo que temer, sino más bien una oportunidad para aprender.

- Evolucionar el prototipo. Las etapas de prueba ayudan a determinar si el prototipo resuelve el problema del usuario. Si no es así, eso no significa fracaso. Más bien, una solución inviable proporciona más información sobre el problema y cómo modificar mejor el diseño.

El proceso de Design Thinking no es lineal, en cualquier momento los estudiantes pueden ir hacia atrás o delante si lo consideran oportuno, saltando incluso a etapas no consecutivas. Se comienza recolectando mucha información, generando una gran cantidad de contenido, que crecerá o disminuirá dependiendo de la fase en la que se encuentren.

Todo ello mediante este proceso iterativo, que utiliza cada una de las etapas en un proceso de tres fases, la experiencia del usuario, el uso de la creatividad y finalmente la ejecución y testeo (Castillo-Vergara, Álvarez-Marín y Cabana-Villca, 2014).

\section{METODOLOGÍA}

Para la determinación metodológica del estudio se tuvo en cuenta que las investigaciones actuales tienden a utilizar modelos dinámicos, flexibles, multidimensionales $\mathrm{y}$, desde luego, sensibles a las características diferenciadas del contexto educativo (Agra, 2005).

Una de las características principales del tema de estudio era abordar el análisis de problemas relacionados con la práctica educativa, por ello se optó por un modelo de investigación etnográfico mediante el método de estudio de caso (Velasco y Díaz de Rada, 2009), muy útiles para la evaluación de aspectos educativos (Woods, 1998).

Como puntualiza Ortega (2005), quien opta por este tipo de estudios entiende la realidad como un conjunto de acontecimientos a los que tiene que acercarse para desvelar las estructuras más profundas y las relaciones que no se ven a simple vista. Además de poder presentar alternativas que mejoren la realidad estudiada mediante el conocimiento de los fenómenos que se analizan.

Siguiendo la propuesta de Losada y López-Feal (2003) la base de la metodología cualitativa estuvo constituida por el trabajo de campo en situaciones naturales durante largo período de tiempo, por ello se consideró optima como método de trabajo.

A través la observación participante se elaboraron datos que luego se analizaron y desde este examen se buscó la generación de conocimientos. Este hecho marcó el sentido de la investigación, ya que se basó en recoger la acción, vivencias y 
experiencias que tuvieron los estudiantes de su realidad. De esta forma se realizó una interacción entre los sujetos que actuaron, que nos ofrecieron la recogida de información y llevó a construir los datos. Es preciso recordar que, debido a que la emergencia fue la base del método de construir teoría, la investigadora no inició el trabajo con una lista de conceptos preconcebidos o un marco teórico cerrado. Tanto los objetivos de la investigación como los núcleos de interés fueron evolucionando paralelamente a la recopilación y análisis de la información.

Siguiendo a Stake (2007), con este estudio se analizó el caso que nos ocupa mediante la descripción del proceso de trabajo y análisis de los problemas que pudieron surgir, así como mediante el estudio de las relaciones establecidas entre los agentes implicados en el caso, para poder alcanzar los objetivos planteados al comienzo del mismo.

\subsection{Contexto de la investigación}

El emplazamiento de campo fue el colegio concertado “Escuelas Pías” de Zaragoza. Este centro educativo se encuentra en un barrio del Casco Antiguo zaragozano, llamado San Pablo, que es considerada una de las zonas más diversas de la ciudad.

El proyecto objeto de estudio se llevó a cabo en el marco de la asignatura de EPVyA, una asignatura obligatoria en el primer ciclo de Educación Secundaria. Tuvo una duración de 14 sesiones, con un total de 28 horas de trabajo durante el tercer trimestre del curso escolar.

Los objetivos recogidos en el proyecto de trabajo eran los siguientes:

- Comprender la aplicación práctica de la metodología en el aula.

- Desarrollar actitudes que favorecieran la asimilación de la metodología por parte del alunado.

- Difundir los resultados de la investigación realizada y de la experiencia vivida por los estudiantes.

Se comenzó con una selección de la muestra que iba a formar parte de la investigación. Al tratarse de un diseño de tipo cualitativo la investigadora decidió a qué personas iba a observar y entrevistar sin necesidad de recurrir a técnicas de muestreo aleatorio. El criterio que se tuvo en cuenta para la elección de la muestra fue la calidad de la información y no la cantidad. En la realización del proyecto participaron 25 estudiantes, 13 alumnas y 12 alumnos. Los sujetos de la investigación estuvieron implicados de una forma natural en el fenómeno de estudio y todos los individuos que participaron lo hicieron de manera activa.

\subsection{Proceso de indagación e instrumentos utilizados}

El proceso de investigación se estructuró en cinco fases (Domínguez, 2015; Rodríguez-Gómez y Valldeoriola, 2009):

- Fase exploratoria, de reflexión y planificación

- Fase de entrada en el escenario (inicio del estudio)

- Fase de recogida y análisis de la información

- Fase de retirada del escenario

- Fase de elaboración del informe de investigación

Se utilizaron diversas técnicas de recogida de datos para facilitar la triangulación. Se realizó recolectándolos desde diversas fuentes de información para su contrastación, considerando las dimensiones temporal y personal (Bisquerra, 2009; Flick, 2014). 
La observación participante se desarrolló, gracias a la colaboración de las familias y del centro escolar, en un entorno real. De este modo la investigadora pudo observar todas las sesiones de trabajo, un total de 14. En las sesiones se recurrió a la observación sistematizada. Se realizaron multitud de notas de campo (descriptivas, textuales y aproximativas) junto con un registro anecdótico. A medida que las ideas teóricas se fueron desarrollando las notas también cambiaron, volviéndose más concretas y detalladas (Hammersley y Atkinson, 1994). Su redacción se realizó de tal manera que proporcionara la información del relato oral y comportamiento no verbal de los participantes, para minimizar el nivel de deducción y facilitar su reconstrucción para su análisis. La observación y posterior análisis de las sesiones fue un pilar fundamental para la elaboración de las preguntas en las diferentes fases del estudio.

Las entrevistas, en la investigación etnográfica, son un instrumento que proporciona datos de primera mano y valiosos, porque ofrecen gran cantidad de información. Como destaca Martínez-Barragán (2011) el lenguaje es a su vez un mundo de categorías calificadas de acuerdo con las sutiles diferencias que hacen los individuos hablantes mientras usan el lenguaje. De aquí que éste, como vehículo y contenido al mismo tiempo, sea unos de los puntos fundamentales de la indagación cualitativa. Al finalizar el proyecto en el aula se realizó una entrevista semiestructurada a los todos los participantes en la investigación. Las preguntas de esta entrevista fueron diseñadas a partir de la organización de un primer sistema de categorías realizado en el proceso de clasificación inicial de los datos.

El grupo de discusión es una técnica empleada comúnmente por investigadores cualitativos (Canales y Peinado, 1994), y también se integra en los diseños de métodos múltiples. La ventaja de este método es que nos permite el análisis de los contextos interactivos en los que se producen las declaraciones y los informes sobre experiencias y acontecimientos (Flick, 2013). Los grupos de discusión se llevaron a cabo en la última fase de la investigación, tras haber transcurrido un año de la realización del proyecto en el aula. Su desarrollo fue similar al de las entrevistas individuales teniendo en cuenta que se buscó una participación igualitaria de todos los participantes. Para lograr esta participación equivalente se optó por organizar los grupos de discusión manteniendo el criterio de grupos de trabajo en que se había desarrollado el proyecto en el aula.

El análisis de documentos se centró en el proyecto de Design Thinking y el portafolio, diarios reflexivos y artefactos realizados por los estudiantes durante el proceso. La realización del portafolio documentó el proceso de enseñar de los profesores y de aprender los estudiantes, pero además les obligó a participar en un proceso abierto que les impulsó a replantear los conocimientos y las estrategias que se fueron adquiriendo y a expresarlos de una manera personalmente significativa.

Como una parte clave del portafolio se realizaron los diarios de reflexión. Al finalizar cada sesión de trabajo el alumnado completó esté documento. Se procuró que los alumnos y alumnas realizaran un trabajo de crítica y reflexión.

Teniendo en cuenta la temática del estudio, se hizo una incidencia especial en el análisis de artefactos: bocetos, dibujos, maquetas y todo material generado por los participantes. Como destaca Gutiérrez (2005) el valor semántico de las representaciones gráficas es un hecho evidente ya que las estructuras gráficas son portadoras de un contenido narrativo, por ello son de gran utilidad como medio para la recogida de información. Por medio del análisis de estos documentos gráficos se buscó tener otro referente de comparación para poder establecer y respaldar algunos datos. 


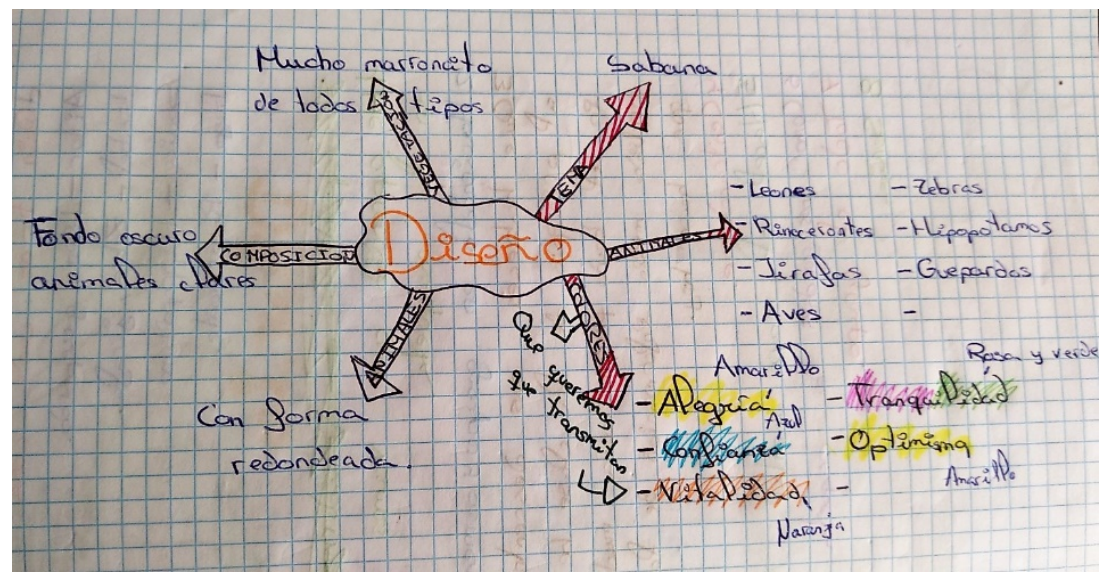

Figura 1. Mapa mental para organizar las ideas iniciales realizado en el portafolio de uno de los sujetos

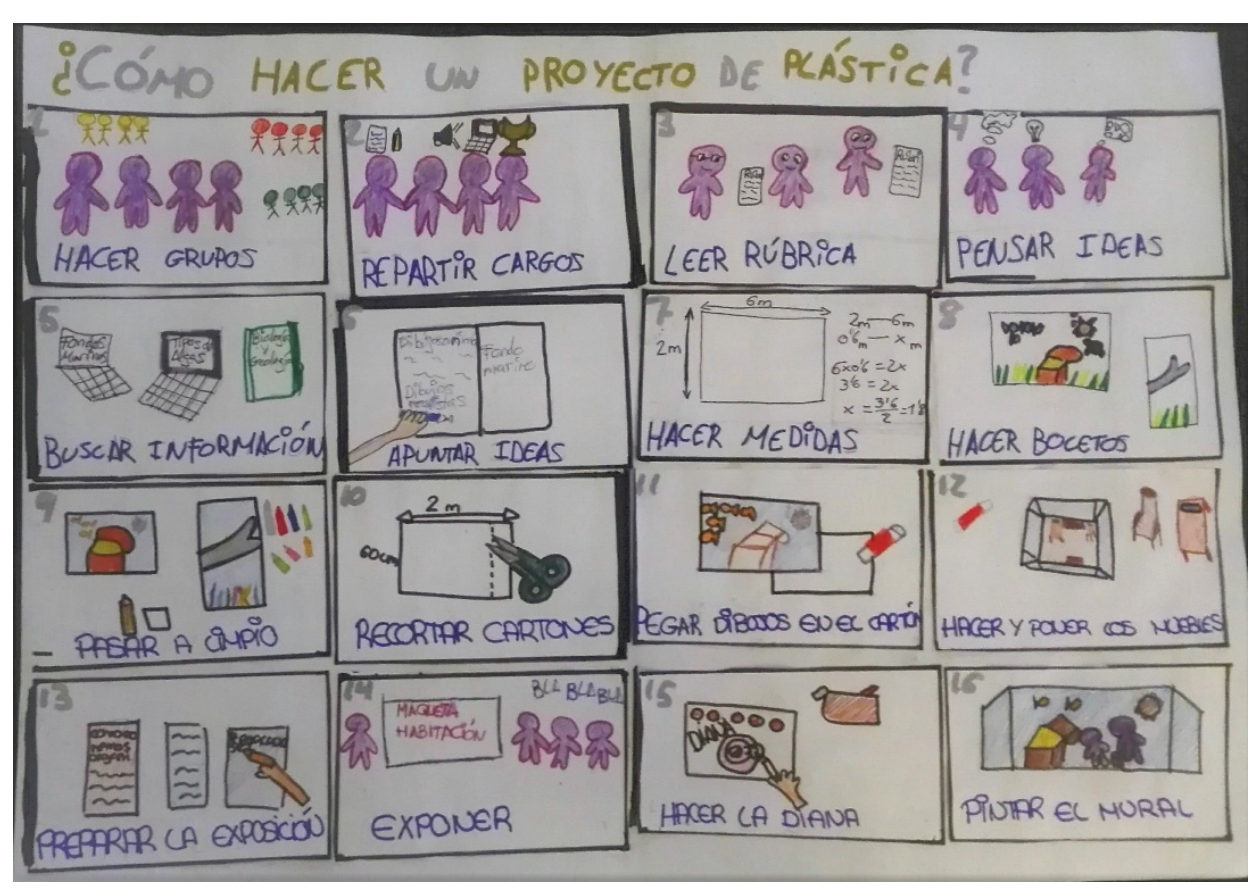

Figura 2. Infografía del proceso de trabajo, realizada por una alumna en el portafolio

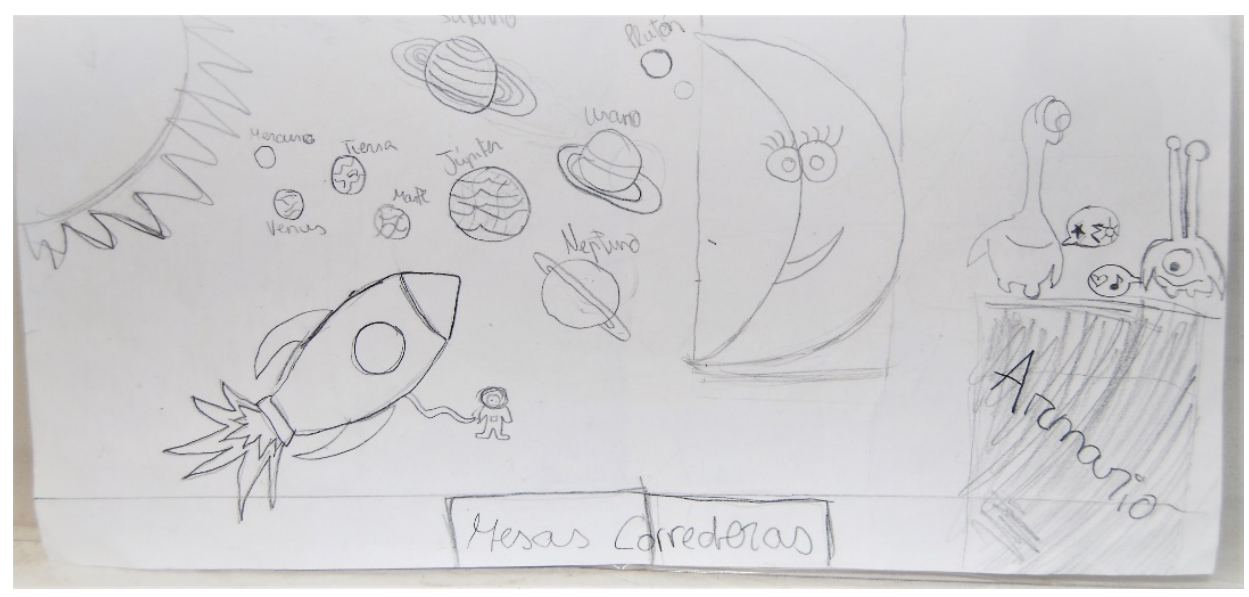

Figura 3. Ejemplo de boceto realizado por el alumnado durante la fase inicial 


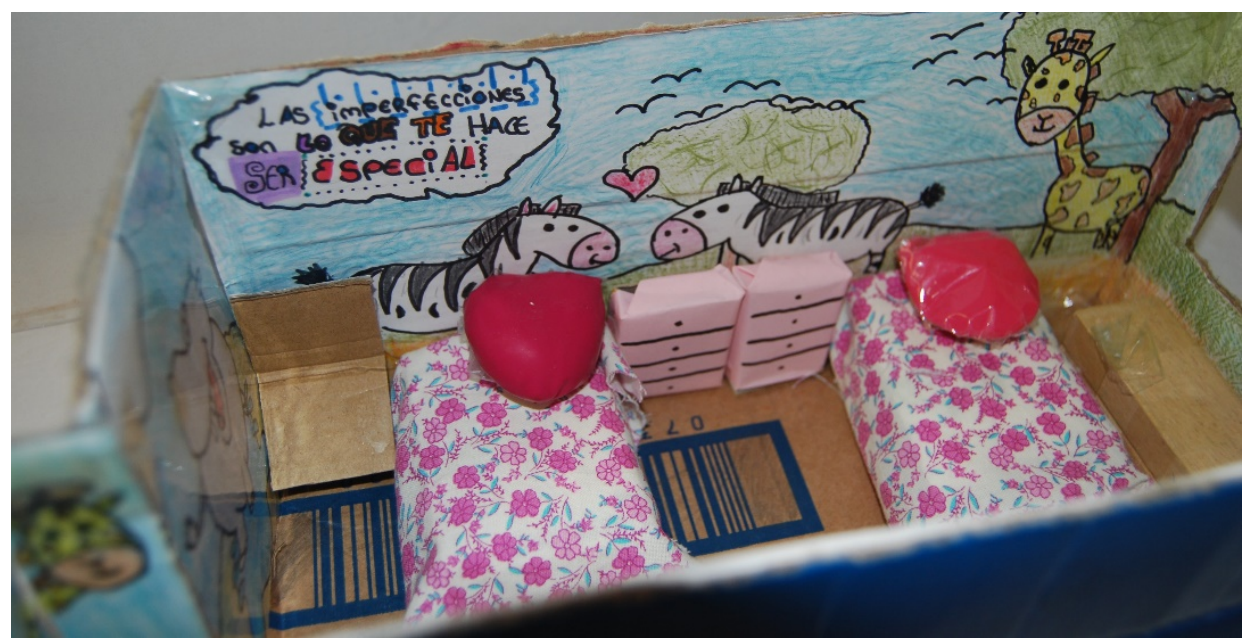

Figura 4. Maqueta realizada por los estudiantes

\subsection{Análisis de datos}

Para el análisis de los datos se siguió el método comparativo constante (Glaser y Strauss, 1967). Este proceso inductivo de análisis de datos abordó la recopilación de información a través de preguntas y notas de campo de los investigadores.

La comparación se realizó dentro del emplazamiento, es decir, se compararon sistemáticamente acontecimientos, situaciones o contextos. Además, se establecieron diferentes tipos de comparaciones en cada una de las etapas del análisis de los datos.

La teoría se desarrolló mediante el registro sistemático de información de campo. No obstante, con anterioridad la investigadora realizó un diario del proceso de codificación y creación de categorías y temas. Para su realización utilizó la técnica que plantea Flick (2012) de realización de preguntas básicas (qué, quién, cómo, cuándo, cuánto, por qué, para qué y por quién), para conseguir el estudio de los textos de manera regular.

Para el análisis de los datos se combinó este proceso con la herramienta de computación ATLAS.ti, ya que permitió la revisión simultánea de una amplia gama de materiales y facilitó su organización y clasificación. Con la ayuda de esta herramienta se buscaron patrones y relaciones entre los datos obtenidos. Durante el proceso de análisis también se elaboraron diagramas, infografías y mapas conceptuales, para facilitar la posterior interpretación de los datos. Con este método, fue posible revelar evidencias y proporcionar una visión más rica de la percepción que las alumnas y alumnos tenían frente sistematización de procesos artísticos gracias a la aplicación de la metodología estudiada.

\section{RESULTADOS}

El objetivo inicial de este estudio fue descubrir la efectividad del modelo metodológico Design Thinking en relación con la mejora del aprendizaje de los contenidos del área de EPVyA. Tras un primer análisis de los documentos generados durante el trabajo de campo se decidió que para conocer esa eficacia se debía valorar el grado de sistematización de los procesos artísticos por parte del alumnado. Para el aprendizaje de una disciplina artística no debemos olvidar la importancia de asimilar los métodos de trabajo relativos a la creación. 
Este apartado se construyó teniendo en cuenta los objetivos del estudio, las fases metodológicas y los temas emergentes que fueron surgiendo a lo largo de la investigación.

\begin{tabular}{cccc}
\hline Categoría & Código & Subcategoría & Código \\
\hline Descubrir & DES & $\begin{array}{c}\text { Inspirar } \\
\text { Empatizar }\end{array}$ & $\begin{array}{c}\text { DESINS } \\
\text { DESEMP }\end{array}$ \\
\hline Idear & IDE & Generar opciones & IDEOPC \\
& & Pensar soluciones & IDESOL \\
\hline Interpretar & INT & Definir problemas & INTDEF \\
& & Analizar argumentos & INTARG \\
\hline \multirow{2}{*}{ Experimentar } & Hacer propuestas & INTPRO \\
\hline Evolucionar & Realizar pruebas & EXPPRU \\
\hline
\end{tabular}

Tabla 1. Categorías, subcategorías y códigos fijados para el análisis

La búsqueda de inspiración para la creación de una obra de arte es el detonante para el inicio de un diseño. En la primera fase del proyecto la búsqueda de información fue una tarea que los grupos de trabajo tuvieron que desempeñar. El objetivo principal fue que el alumnado aprendiera a recopilar datos para la realización de sus diseños.

Antes de comenzar con esta tarea, se les motivó para que realizaran una reflexión sobre sus necesidades y posibles métodos de búsqueda.

Durante este proceso el alumnado tuvo presente su experiencia vital. Por ejemplo, un estudiante en la segunda sesión de trabajo explicó: "Me acordé de una cosa de Lego que vi en una habitación y busqué cosas parecidas”.

Los participantes en el estudio se dieron cuenta de la importancia de realizar el proceso de investigación de forma correcta para poder tener referentes visuales en los cuales basar sus diseños. Al respecto de esta cuestión, una alumna destacó en la sesión sexta de trabajo: “Aprendí que tenemos que tener presente siempre una imagen o algo para guiarnos para hacer el boceto. He aprendido a cómo dibujar bien los animales. Lo he aprendido preguntando a la profesora y mirando el libro. Me ha costado hacer la composición, he tenido que mirar el libro e investigar”.

Durante esta fase fue fundamental que los estudiantes conectaran con los usuarios a los que estaba destinado su diseño. La generación de afinidad es un aspecto necesario dentro del proceso de cualquier creación artística. Los alumnos y alumnas tuvieron que reflexionar sobre las necesidades y preferencias del colectivo al que el diseño fue destinado. En este orden de ideas, una alumna planteó en los grupos de discusión: "Entre todos decidimos lo que sería mejor. Lo que nos gustaría ver a nosotros si estuviéramos allí”

Para el análisis de las diversas propuestas de los miembros del grupo se utilizaron los mapas mentales. Se seleccionó esta herramienta ya que tiene una gran carga visual al organizar las ideas en forma de diagrama. Este ejercicio les ayudó a generar opciones, pensar soluciones, concretar ideas y registrar los acuerdos. Inicialmente no entendieron el objetivo de esta tarea: "Hemos hecho el mapa mental y ya no sabemos qué hacer".

Tras una fase de análisis comprendieron su utilidad para la organización de las ideas. Todos los grupos manifestaron que había sido una buena herramienta para compartir las ideas, actividad que les solía costar mucho esfuerzo. Una alumna planteó en la cuarta sesión: "Hoy mi grupo y yo hemos empezado a hacer el mapa mental. Hemos aprendido 
a poner en común ideas y, aunque nos ha costado bastante elegir el resultado final, pero lo hemos superado poniendo todas las ideas en común”.

Una vez se definieron las temáticas de trabajo de cada grupo se inició la fase de realización de bocetos. La generación de opciones no la efectuaron de manera correcta. Un alumno explicó en la entrevista: “Creo que hemos improvisado. Encontramos ideas comunes que nos gustaron, pero luego no las usamos para los bocetos. Poníamos lo que nos gustaba a cada uno y punto. Empezamos bien, buscando información y todo eso, tuvimos muchas ideas, pero luego se fueron quedando todas atrás y nos atascamos mucho con lo de dibujar”.

El alumnado no logró realizar una definición correcta del problema ya que no analizaron los argumentos expuestos por cada miembro del equipo. A pesar de ello, los estudiantes comprendieron la función dentro del proceso creativo de la realización de bocetos y por ello mostraron interés por su aplicación y aprendizaje.

El proceso manipulativo para la realización del prototipo fue muy relevante para todos los participantes, posiblemente porque nunca habían realizado una maqueta. En la entrevista fue considerado un elemento significativo en el aprendizaje de contenidos de la asignatura. No debemos olvidar que los contenidos para trabajar en el proyecto, aunque se seleccionaron por parte del docente en el diseño inicial, se fueron estableciendo con relación a las necesidades que los estudiantes presentaron a nivel conceptual para la resolución de los problemas. Por ello, en muchas ocasiones se trabajaron contenidos curriculares de segundo ciclo de Secundaria.

La falta de definición argumentada en las primeras fases del proyecto provocó que las ideas finales fueran asumidas sin someterlas a análisis. Todos los grupos se quedaron con la primera idea de diseño sin plantear más opciones. Una alumna explicó en la última sesión de trabajo: “Podríamos volver a repasar las ideas anteriores para ver las ideas que tuvimos buenas en los procesos de descubrir e interpretar. Teníamos buenas ideas al principio, pero luego empezamos a bloquearnos porque esa idea nos daba problemas y a no ponernos de acuerdo. Entonces dejamos de lado las ideas que habíamos buscado y empezamos a hacer lo mismo que otros grupos, cuando la otra creo que era mucho mejor”.

Al finalizar el proceso los estudiantes fueron capaces de evaluar las fuentes de donde habían extraído la información: "En internet también hay información falsa que te puede mostrar información que no te interesa o no hayas puesto en el buscador, hay que diferenciar la información falsa (...) En internet hay mucha información basura, no sólo te tienes que fiar de la primera página que te muestra el buscador”.

Durante el proceso de trabajo el alumnado fue capaz de identificar sus dificultades para poder superarlas y transformarlas en habilidades académicas. En los grupos de discusión se destacó la adquisición del aprendizaje gracias a los errores: "He aprendido de nuestro error principal que ha sido no organizarnos, no organizarnos bien. Con este método se te queda en la cabeza la experiencia y aprendes de los errores y esa hace que se te quede en la cabeza”. También se incidió en el aumento de los momentos de reflexión sobre las tomas de decisiones durante el transcurso del proyecto: "Al principio la verdad que hicimos todo de forma más impulsiva, entonces fuimos avanzando en el trabajo y si reflexionamos más y al acabar sí que sacamos, por así decirlo, una conclusión”. 


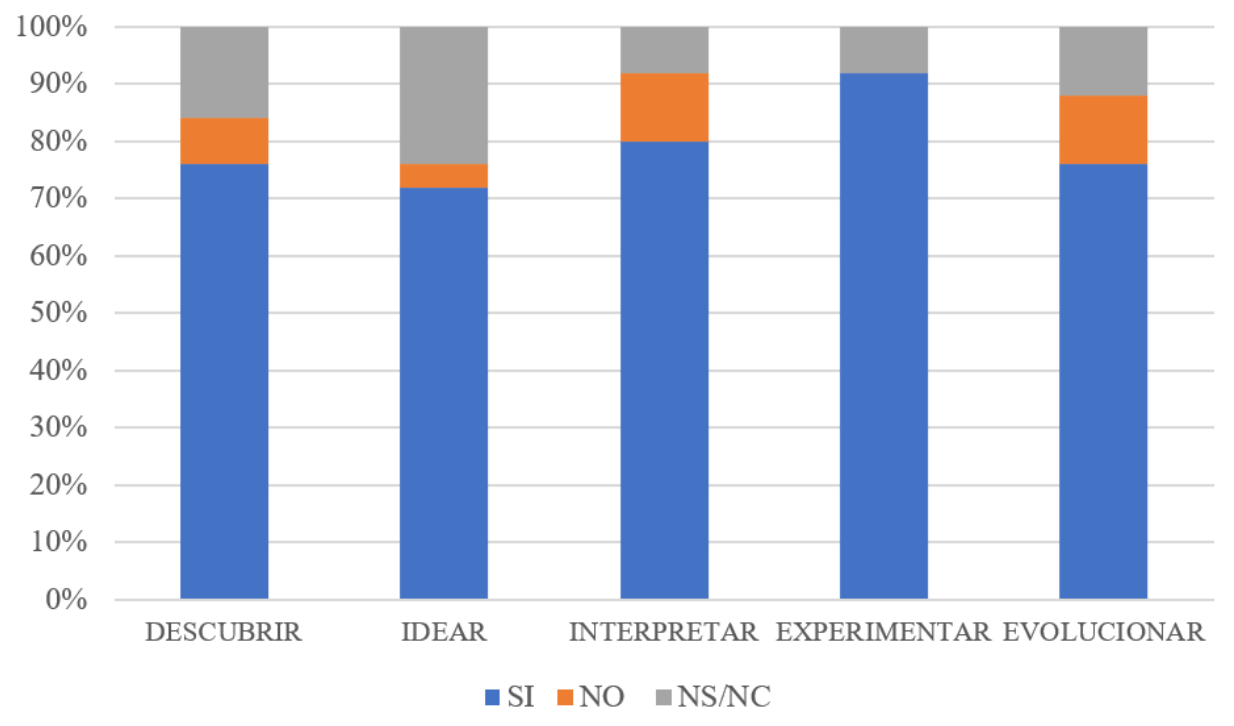

Tabla 2. Percepción de los estudiantes en la entrevista sobre la sistematización de procesos artísticos

\section{DISCUSIÓN Y CONCLUSIONES}

La actividad artística se fundamenta principalmente en el desarrollo práctico de los contenidos, por ello se considera oportuno organizar el área centrándose en la experimentación activa, donde por medio del proyecto el alumnado aprenda haciendo. Podemos afirmar que, al igual que lo planteado por Rodríguez (2017), los estudiantes aprendieron a través de investigación activa, el desarrollo práctico y el diseño participativo.

Autores como Araño (2005) consideraron que el hecho de que los aprendizajes sean significativos debe ser una de las características esenciales del modelo cognitivo de estructuración de la práctica para la educación artística. Esta idea se sustenta en considerar el área de educación artística como especificidad en el ámbito de construcción de significados a través prioritariamente de la imagen visual, con un interés destacado por las producciones artísticas y de cultura visual (Barragán, 2005).

Una formación artística crítica e integradora debería realizarse mediante aprendizaje estratégico: poner menos énfasis en los contenidos y más en estrategias de aprendizaje (Barragán, 2005). Por medio del desarrollo del Design Thinking se debe proyectar, ejecutar y evaluar, aspectos relevantes para desarrollar la competencia de aprender a aprender.

Al igual que Da Silva (2018), se observó que el aprendizaje activo es especialmente adecuado cuando se desarrollan actividades relacionadas con la práctica del diseño, debido a la necesidad que tiene esta disciplina artística en garantizar un producto final con una función específica. De forma similar que el estudio de Ulger (2018), podemos decir que los estudiantes dedicaron más esfuerzo en las etapas de pensamiento y diseño de la obra que mediante métodos de enseñanza tradicional. Además, comprendieron la necesidad de tomar referentes visuales para poder sustentar sus ideas de forma gráfica.

Como destaca García-Sípido (2005) el aprendizaje plástico no debe ser un conjunto de contenidos ya sabidos o verdades ya descubiertas. La investigación es un proceso clave dentro de la plástica, y por ello los procesos de investigación son claramente 
definitorios del área. El alumnado no siempre es capaz de sistematizar este proceso para una verdadera operatividad de los contenidos y significación en sus aprendizajes. Los rasgos que caracterizan a un proyecto creador, según Marina (1994), son la libertad, la creación, la inteligencia humana y la creatividad, y el Design Thinking busca el desarrollo coherente de habilidades de aprendizajes creativos e independientes (Klein, Taveras, King, Commitante, Curtis-Bey y Stripling, 2009).

Las metodologías activas dirigen a los estudiantes a investigar ideas y preguntas importantes y está enmarcado en un proceso de investigación. Se diferencia de acuerdo con las necesidades e intereses de los estudiantes (Klein et al., 2009).

$\mathrm{Al}$ igual que en el estudio presentado por Thomas (2000) debemos destacar que los alumnos y alumnas presentaron ciertas dificultades al afrontar la investigación con un alto grado de autonomía, principalmente al inicio de la investigación y la gestión del tiempo. La efectividad de esta metodología como método de aprendizaje vino dada por la incorporación de apoyos a los estudiantes para aprender a cómo utilizarla.

Una de las razones por la que se dispersaron fue el no saber manejar la cantidad de información que habían acumulado; al carecer de herramientas para procesarla se produjo en algunos casos el abandono de la tarea para dar paso a las conversaciones y distracciones. Aunque se estimuló la utilización de esquemas, cuadros sinópticos y mapas conceptuales, pocos grupos aprovecharon estas estrategias (López y Lacueva, 2007). El uso de estas herramientas facilitó el metaaprendizaje y metaconocimiento (Mendioroz, 2016) pero consideramos que el nivel madurativo del alumnado de Secundaria no es suficiente para que favorezca la adquisición de conocimiento.

Al igual que Pihl (2015) destacamos la importancia de compartir entre los integrantes del grupo el proceso creativo. Para que este proceso sea efectivo se debe crear un flujo constante de creación de bocetos, imágenes y maquetas. Cada una de las propuestas debe ser seguida de una reflexión en grupo donde se compartan las experiencias individuales del desarrollo productivo.

Podemos concluir diciendo que, a pesar de las dificultades, el Desing Thinking permitió a los estudiantes desarrollar habilidades y metodologías de investigación para resolver problemas y conocer qué recursos eran útiles, qué estrategias eran más efectivas y qué podían hacer para que su trabajo funcionara mejor. Para que el aprendizaje fuera efectivo durante la fase final del proyecto los estudiantes necesitaron comprender la necesidad de aplicar habilidades de reflexión.

Esta metodología trabajó la mejora de habilidades para la búsqueda de información, elaboración de conclusiones y creación de contenido. A pesar de ello fue complicado que los estudiantes se involucraran en el proceso de investigación constructiva. Les costó desarrollar habilidades de aprendizaje creativo e independiente.

Por medio de este modelo didáctico los alumnos aprendieron a manejar información, filtrarla, codificarla, categorizarla, evaluarla, comprenderla y utilizarla. Mediante la realización del proyecto tuvieron que proyectar, ejecutar y evaluar, aspectos relevantes para desarrollar la competencia de aprender a aprender. Se logró que comprendieran la importancia de gestionar el conocimiento para resolver situaciones creativamente y no ser meros reproductores de información.

Los estudiantes aprendieron de sus errores, aunque durante el desarrollo del proyecto les costó asimilar muchos aspectos relacionados con la autorregulación de su aprendizaje y planificación del trabajo. 
Esta metodología permitió al estudiante detectar las necesidades para comprender mejor el problema, identificar principios que respaldan el conocimiento y cumplir objetivos de aprendizaje relacionados con cada parte del programa de la asignatura.

En resumen, en cuanto a la efectividad de este modelo metodológico, podemos decir que mejoró en el estudiante su capacidad para sistematizar el proceso de creación artística por medio de la comprensión de la operatividad de los contenidos.

\section{BIBLIOGRAFÍA}

Acaso, M. (2009). La educación artística no son manualidades. Nuevas prácticas en la enseñanza de las artes y la cultura visual. Madrid: Catarata.

Acaso, M. y Megías, C. (2017). Art Thinking. Cómo el arte puede transformar la educación. Barcelona: Paidós Educación.

Aflatoony, L., Wakkary, R. y Neustaedter, C. (2017). Becoming a Design Thinker: Assessing the Learning Process of Students in a Secondary Level Design Thinking Course. The international Journal of Art and Design Education, 37 (3), 438-453.

Agra, M. (2005). El vuelo de la mariposa: la investigación artístico-narrativa como herramienta de formación. En R. Marín-Viadel. Investigación en Educación Artística (pp. 126-150). Granada: Universidad de Granada.

Araño, J. (2005). Estructura del conocimiento artístico. En R. Marín-Viadel. Investigación en Educación Artística (pp. 17-42). Granada: Universidad de Granada.

Arbizu, F. (1994). La función docente del profesor universitario. Bilbao: Universidad del País Vasco.

Barragán, J. (2005). Educación artística, perspectivas críticas y práctica educativa. En R. MarínViadel. Investigación en educación artística (pp. 41-80). Granada: Universidad de Granada.

Bisquerra, R. (2009). Metodología de la investigación educativa. Madrid: La Muralla.

Calaf, R. y Fontal, O. (2010). Cómo enseñar arte en la escuela. Madrid: Editorial Síntesis.

Canales, M. y Peinado, A. (1994). Grupos de discusión. En J. Delgado y J. Gutiérrez. Métodos y técnicas de investigación en ciencias sociales (pp. 287-316). Madrid: Síntesis.

Castillo-Vergara, M., Álvarez-Marín, A. y Cabana-Villca, R. (2014). Design Thinking: como guiar a estudiantes, emprendedores y empresarios en su aplicación. Ingeniería Industrial, 35 (3), 301-311.

Coll, C. (1978). La conducta experimental en el niño. Barcelona: Ediciones CEAC.

Da Silva, A.A. (2018). Interactive Art, Performance and Scientific Research into Corporeal Empathy. Journal of Problem Based Learning in Higher Education, 6 (1), 39-54.

Domínguez Montes, J. (2015). De la investigación científica a la investigación cualitativa en educación. Revista Supervisión 21, 36, 1-22.

Flick, U. (2012). Introducción a la colección: Investigación cualitativa. En M. Angrosino Cualitativa, Etnografía y observación participante en investigación (pp. 9-13). Madrid: Morata.

Flick, U. (2012). Introducción a la investigación cualitativa. Madrid: Morata.

Flick, U. (2013). Sobre este libro. En R. Barbour. Los grupos de discusión en Investigación Cualitativa (pp. 21-22). Madrid: Morata.

Flick, U. (2014). La gestión de la calidad en Investigación Cualitativa. Madrid: Morata.

Fontal, O., Gómez, C. y Pérez, S. (2015). Didáctica de las artes visuales en la infancia. Madrid: Ediciones Paraninfo.

Fontal, O., Marín, S. y García, S. (2015). Educación de las artes visuales y plásticas en educación primaria. Madrid: Ediciones Paraninfo.

García-Sípido, A. (2005). La investigación plástica como método paradigmático de conocimiento. En R. Marín-Viadel. Investigación en Educación Artística (pp. 81-98). Granada: Universidad de Granada. 
Glaser, B.G. y Strauss, A.L. (1967). The discovery of grounded theory. Chicago: Aldine.

Gutiérrez, R. (2005). Los estudios de casos: una opción metodológica para investigar la educación artística. En R. Marín-Viadel. Investigación en Educación Artística (pp. 151-174). Granada: Universidad de Granada.

Hammersley, M. y Atkinson, P. (1994). Etnografía. Métodos de investigación. Barcelona: Paidós Básica.

Hernández, F. (1995). El diseño curricular de educación visual y plástica: un análisis crítico. Revista Interuniversitaria de Formación del Profesorado, 24, 21-37.

Hernández, F., Jódar, A. y Marín, R. (1991). Antes del recorrido, ¿por qué un libro sobre la educación artística? En F. Hernández, A. Jódar y R. Marín. ¿Qué es la educación artística? (pp. 13-18). L'Hospitalet de Llobregat: Sendai.

Klein, J., Taveras, S., King, E.D., Commitante, A., Curtis-Bey, L. y Stripling, B. (2009). ProjectBased Learning: Inspiring Middle School Students to Engage in Deep and Active Learning. Nueva York: NYC Department of Education.

López, A.M. y Lacueva, A. (2007). Enseñanza por proyectos: una investigación-acción en sexto grado. Revista de Educación, 342, 579-604.

Losada, J. y López-Feal, R. (2003). Métodos de investigación en Ciencias Humanas y Sociales. Madrid: Thomson.

Marina, J. (1994). Teoría de la inteligencia creadora. Barcelona: Anagrama.

Martínez-Barragán, C. (2011). Metodología cualitativa aplicada a las Bellas Artes. Revista Electrónica de Investigación, Docencia y Creatividad, 1, 46-62.

Mendioroz, A. (2016). Empleo de herramientas metacognitivas para realizar el proyecto final de licenciatura en la Escuela de Ingeniería ESIME-Culhuacán, del Instituto Politécnico Nacional de México. Publicaciones de la Universidad de Murcia, 31 (1), 197-221.

Nisbet, J. y Shuckmith, J. (1980). Estrategias de aprendizaje. Madrid: Santillana.

Ortega, A. (2005). El estudio de caso: implicaciones epistemológicas de un método de investigación aplicado a la educación artística. En R. Marín-Viadel. Investigación en Educación Artística (pp. 295-322). Granada: Universidad de Granada.

Pellicer, C., Álvarez, B. y Torrejón, J. (2013). Aprender a emprender: cómo educar el talento emprendedor. Girona: Fundación Príncipe de Girona, Aulaplaneta.

Pihl, O. (2015). Hidden Realities inside PBL Design Processes: Is Consensus Design an Impossible Clash of Interest between the Individual and the Collective, and Is Architecture Its First Victim? Journal of Problem Based Learning in Higher Education, 3 (1), 20-45.

Portnoy, L. (2018). Design Thinking Adds Why to the What and How of Learning. Thinking Like a Designer. 13 (15), 1-5.

Rodríguez, C.M. (2017). A Method for Experiential Learning and Significant Learning in Architectural Education via Live Projects. Arts and Humanities in Higher Education: An International Journal of Theory, Research and Practice, 17 (1), 279-304.

Rodríguez-Gómez, D. y Valldeoriola, J. (2009). Metodología de la investigación. Barcelona: Universitat Oberta de Cataluña.

Sánchez, S., Brahim, C. y López, V. (2013). El proceso de aprendizaje a través de un pensamiento complejo. Innovation in Engineering, Technology and Education for Competitiveness and Prosperity (pp. 1-8). Cancún: Eleventh LACCEI Latin American and Caribbean Conference for Engineering and Technology (LACCEI'2013).

Serrano, M. y Blázquez, P. (2014). Design thinking: Lidera el presente. Crea el futuro. Madrid: ESIC Editorial.

Stake, R. (2007). Investigación con estudio de casos. Madrid: Morata.

Steinbeck, R. (2011). Building Creative Competence in Globally Distributed Courses through Design Thinking. Comunicar, 37 (19), 27-35.

Thomas, J.W. (2000). A review of research on project-based learning. California: The Autodesk Foundation. 
Ulger, K. (2018). The Effect of Problem-Based Learning on the Creative Thinking and Critical Thinking Disposition of Students in Visual Arts Education. Interdisciplinary Journal of Problem-Based Learning, 12 (1), 1-12.

Velasco, H. y Díaz de Rada, A. (2006). La lógica investigación etnográfica. Un modelo de trabajo para etnógrafos de escuela. Madrid: Trolla.

Woods, P. (1998). La escuela por dentro. La etnografía de la investigación educativa. Barcelona: Paidós/MEC. 